\title{
Kriterien für die Bestimmung der Zugehörigkeit des Islam zu Deutschland
}

Klaus Spenlen und Norbert Posse

\section{Theoretische Annäherung an „Zugehörigkeit“ Klaus Spenlen}

Wohl jeder kennt den Ausruf „Gib her, das gehört mir!“ oder die Frage „Wem gehört das?". Die meisten dieser Besitz- oder Eigentumsfragen sind zumindest dann relativ leicht zu beantworten, wenn bei Eigentumsansprüchen Dokumente vorgelegt werden können wie Kaufbelege, Schenkungsurkunden oder Grundbucheinträge. Nicht viel schwerer stellen sich Zuordnungen bei einer Ankündigung wie dieser dar: „Für Angehörige der Kunstakademie ist der Eintritt frei“. Eine Angehörigkeit ist durchweg ebenfalls ein Rechtsverhältnis wie das zu Besitz oder Eigentum, das sich allerdings in einer Mitgliedschaft, einem Arbeitsverhältnis o. Ä. begründet. Hier kann der Beweis der Angehörigkeit z. B. durch Vorlage eines Mitglieds- oder Dienstausweises erbracht werden.

Schwieriger ist die Frage der Zugehörigkeit zu beantworten, z. B. ob etwa Regelverstöße zum Straßenverkehr gehören. Im Vergleich zu den Besitz- oder Eigentums- sowie Angehörigkeitsfragen macht dieses Beispiel viererlei deutlich: Erstens gibt es offenbar einen Unterschied zwischen Rechtsverhältnissen und solchen, die sich auf Zugehörigkeit berufen. Zweitens bedeutet Zugehörigkeit nicht in jedem Fall Machtanspruch, gar Verfügungsgewalt über etwas oder jemanden zu haben. Drittens scheint es Zugehörigkeiten zu geben, die beobachtbar, fotografierbar, protokollierbar oder zählbar sind, denn Übertretungen im Straßenverkehr kennt jeder aus eigener Anschauung, im Zweifel aus Fotos von Radareinrichtungen. Viertens scheint es Zugehörigkeiten selbst bei Sachverhalten zu geben, bei denen sie nicht von vornherein zu erwarten sind: So wie etwa Regelverstöße zum Straßenverkehr gehören, gehört der Tod zum Leben. Jemand oder etwas kann sich mithin zugehörig fühlen, ohne dabei angehörig zu 
sein oder Besitz zu beanspruchen. Einem Staat wie Deutschland, der eine politische Ordnung mit entsprechender rechtlicher und gesellschaftlicher Struktur besitzt, können mithin auch Menschen angehören, indem sie hier wohnen oder die Staatsangehörigkeit besitzen, ohne sich dem Volk zugehörig zu fühlen, dessen Vorfahren ihn gegründet haben. Um Zugehörigkeit zu reklamieren, bedarf es also keines einklagbaren Rechtstitels, eher Emotionalität, Verbundenheit, Wir-Gefühl. Es kann aber ggf. hilfreich sein, bei Bedarf weitere Informationen hinzuzuziehen, ob etwas zugehörig ist, etwa Statistiken, Nachrichten oder anderweitiges Datenmaterial.

Das lässt sich z. B. an der Frage erläutern, ob Migranten zu Deutschland gehören. Hier helfen Beobachtungen oder Befragungen zwar weiter. Zusätzlich ist jedoch ein Blick in die Geschichte hilfreich, denn Aus- und Zuwanderung hat es schon immer gegeben, sie sind so alt wie die Menschheit selbst. Mithin ist selbst dann die Zugehörigkeit zur neuen Lebensumgebung nicht zweifelhaft, wenn sie auf gängige Aussagen von Migranten wie „... ist halt beides irgendwie meine Heimat", das Alte und das Neue, stößt. ${ }^{1}$

Beobachtbar sind in Deutschland auch Muslima, die sich islamisch kleiden, also beispielsweise ihr Haar mit einem Kopftuch bedecken. Auch bei manchen Männern kann man eine islamische Kopfbedeckung, die Tacke, sehen. Hilft hier als weitere Information der Blick in deren Pass? Gehören Menschen islamischen Glaubens dann zu Deutschland, wenn sie hier geboren sind und die deutsche Staatsangehörigkeit besitzen? Und anders herum gefragt: Gehören sie nicht zu Deutschland, wenn diese Merkmale nicht auf sie zutreffen? Geht es bei der Frage nach ihrer Zugehörigkeit zu Deutschland also um die Person oder um schwer fassbare Kennzeichen wie deren Glaube und Religion?

Eine der Grundfragen dieses Beitrags ist mithin die Frage, ob „der“ Islam in Deutschland die Summe aller Muslime ist, die hier leben oder ob er eine eigene, beschreibbare und bestimmbare Qualität darstellt.

Bei dieser Qualitätsfrage geht es zunächst darum, zu klären, was „der“ Islam ist. Die derzeit etwa vier Millionen Muslime in Deutschland gehören, sofern sie

\footnotetext{
${ }^{1}$ Eine im August 2012 durchgeführte Umfrage des Instituts Info GmbH unter insgesamt 1.011 Deutsch-Türken bestätigt solche Einstellungen und Gefühle von der ersten bis zur dritten Generation der Türkischstämmigen, zumeist Muslimen. Das Ergebnis: Viele von ihnen fühlen sich zwischen beiden Kulturen „zerrissen“.
} 
sich zum Islam bekennen und gläubig sind, einer der zahlreichen islamischen Organisationen und Strömungen unterschiedlicher Schattierungen an. Dazu gehören die großen Richtungen der Sunniten, Aleviten, Schiiten, aber auch Minderheiten wie die Ahmadiyya sowie fundamentalistische Strömungen wie die (Neo-)Salafisten, die sich bekannten Organisationsmustern weitgehend entziehen. ${ }^{2}$ Sofern der Islam als Religion verstanden wird, gehört er zu den z. B. für Nordrhein-Westfalen ermittelten 228 religiösen Gemeinschaften und Strömungen. $^{3}$

Ob er damit zugleich zu Nordrhein-Westfalen (oder Deutschland) gehört, könnte nicht zuletzt von seiner Vereinbarkeit mit dem deutschen Verfassungsstaat abhängen. Dazu gehört der Sachverhalt, dass sich „der“ Islam nicht nur als Religion, sondern auch als Staatstheorie versteht. ${ }^{4}$ Kann eine abweichende Staatstheorie rechtmäßig zu einem demokratischen, pluralistischen und rechtstaatlichen Staat wie Deutschland nach Art. 20 GG auch dann gehören, wenn sie die Ablehnung von Verfassungsgrundlagen dieses Staates beinhaltet? Aus dieser Grundsatzfrage ergeben sich zahlreiche verfassungsrechtliche Einzelfragen wie diejenige, ob die Basis des Islam, also Koran und Sunna (Hadîthe), z. B. die positive und negative Religionsfreiheit (Art. 4 GG), die Gleichstellung von Mann und Frau (Art. 3 GG) sowie uneingeschränkt alle weiteren Grundrechte anerkennt, ${ }^{5}$ die durch das Grundgesetz und die Landesverfassungen garantiert werden. Diese grundlegenden Freiheits-, Gleichheits- und Unverletzlichkeitsrechte stehen in Deutschland dem Einzelnen gegenüber dem Staat zu, aber auch gegenüber der Gesellschaft (Art. 1-17, 33, 101-104 GG). Die meisten dieser Grundrechte sind zugleich Menschenrechte, das bedeutet, nicht nur deutsche Staatsbürger können sich auf sie berufen, sondern alle Menschen, die in Deutschland leben. ${ }^{6}$

\footnotetext{
${ }^{2}$ Vgl. Volkhard Krech 2009, S. 10 ff. sowie die Beiträge von Hayrettin Aydın (S. 105-120) und Ekkehard Rudolph (S. 191-209) in diesem Sammelband.

${ }^{3}$ Vgl. Markus Hero u. a. 2008.

${ }^{4}$ Vgl. Younes Nourbakhsh 2008.

${ }^{5}$ Die feinsinnige Unterscheidung, die manche Verbandsmuslime in Kommunikationsforen wie etwa der Deutschen Islamkonferenz wiederholt betonen, die Grundrechte zwar akzeptieren, sie aber nicht anerkennen zu wollen, wirft den Diskurs über die Grundsatzfrage eher zurück, als dass sie ihn weiterbringt.

${ }^{6}$ Vgl. zu dem Bereich „Islam und Verfassungsstaat“ den Beitrag von Wolfgang Bock (S. 163-190) in diesem Sammelband.
} 
Über die Beantwortung verfassungsrechtlicher Fragen hinaus ergeben sich Fragen, was über das Grundgesetz sowie über das Staatsgebiet hinaus Deutschland ausmacht. Zweifellos ist das heutige Deutschland bestimmt durch seine völkerrechtliche und europäische Identität sowie eine staatsrechtliche Kontinuität. Diese Kennzeichen beinhalten zugleich unveräußerliche Werte, die sich unabhängig von ökonomischen Werten herausgebildet haben.

Da Gesellschaften „Kommunikationseinheiten“ sind, ${ }^{7}$ werden zwangsläufig und regelmäßig Kommunikationshürden sichtbar, die allerdings ganz unterschiedlich markiert sein können. Eine Hürde kann die des ethnischen und des ethno-nationalen Markers sein. Ethnizität versteht sich dann als ein europäisches Konzept von sozialer und politischer Gemeinschaftlichkeit, mithin mit nationalistischer Konnotation. „Nationalismus stellt jedenfalls die nationale Identität als vorrangige soziale Identität in das Zentrum politischer Überlegungen. Diese fasst er als ethnische Identität auf. Daher tritt er bis nahe an die Gegenwart immer als Ethno-Nationalismus auf."

Marker im hier diskutierten Kontext sind z. B. gemeinsame Geschichte, Sprache, eine „gewachsene“ Religionslandschaft und eben (unveräußerliche) Werte. Also scheint es bei der Zugehörigkeit des Islam zu Deutschland auch um die Frage zu gehen, ob der Kern dessen, was Deutschland ausmacht, durch die $\mathrm{Zu}-$ gehörigkeit des Islam zu Deutschland berührt, gar infrage gestellt wird. Diese Frage nimmt als eigentliches soziales Integrationsproblem des Islam die Grenzziehung zwischen Gesellschaften („Deutschland“, „Deutsche“) sowie Gemeinschaften und Gruppen („Islam“, „Muslime“) vor. Wie aber kann dennoch Gemeinschaftlichkeit, gar Zugehörigkeit zustande kommen? Nach den bisherigen Ausführungen muss das Merkmal „Ähnlichkeiten von Menschen untereinander" ausgeschlossen werden, weil kin selection, die sozio-kulturelle Zugehörigkeit durch Ähnlichkeitsmerkmale kennzeichnet, mithin andere als stereotype Deutsche, also etwa Muslime oder „den“ Islam, ausgrenzt. Eine andere Erklärung gründet in der „[...] der Kultur [...], etwa im Sinne des ,Kulturkreises‘. Dieser fiel früher im Okzident, und heute noch bei vielen Menschen, mit der Re-

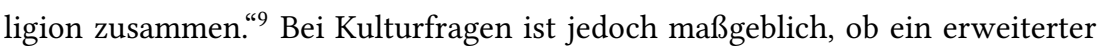

\footnotetext{
${ }^{7}$ Vgl. Karl Wolfgang Deutsch 1953.

${ }^{8}$ Albert F. Reiterer 2012, S. 29.

${ }^{9}$ Ebenda, S. 25.
} 
lebensweltlich orientierter oder ein enger Kulturbegriff zugrunde gelegt wird. ${ }^{10}$ Der erweiterte und pragmatische Kulturbegriff verlässt das angesprochene dominante Zugehörigkeitsmerkmal der Religion und öffnet sich weiteren Eigenschaften.

Will man bei unserer Frage zudem die Ebene politisch-ideologischer Theorien verlassen und zu „lebensweltlicher Orientierung“ gelangen, hilft der Begriff der Identität weiter.

Identität [...] ist ein Prozess des Aufbaus von Sinn und Bedeutung auf Grundlage kultureller Eigenschaften, denen man den Vorzug vor anderen Sinnbezügen gibt. Für ein Individuum oder einen sozial Handelnden kann es eine Vielzahl von Identitäten geben. Aber das ist eine Quelle von Stress in der Selbstdarstellung wie auch im Handeln. [...] Denn im Vergleich zu Rollen sind Identitäten Ergebnis eines tiefer greifenden Prozesses. [...] Identitäten legen den Sinn fest, während Rollen nur die Funktion definieren. [...] Sinn wird um die Primäridentitäten herum organisiert (um solche also, welche die anderen Identitäten stützen). ${ }^{11}$

Identität entsteht dadurch, dass Personen sich über Zugehörigkeiten und Nicht-Zugehörigkeiten definieren, die ihr Alltagshandeln bestimmen. So baut sich auch ihre persönliche Identität aus unterschiedlichen sozialen Identitäten auf und erschwert damit den Aufbau einer Identität mit Exklusivitätsanspruch, etwa der der nationalen Identität. Aber gerade durch das Vorhandensein multipler Identitäten kann es für den Einzelnen zu Loyalitätskonflikten konkurrierender Identitäten kommen, die jeweils normative Ansprüche erheben. D. h., wenn sich Muslime gleichermaßen mit Deutschland und „dem“ Islam identifizieren wollen, müssen sie ihre Religion widerspruchsfrei zu ihrem Leben in Deutschland machen, beide Bereiche mithin harmonisieren. Und - bei einer Umkehrung der Perspektive in der Zugehörigkeitsfrage - wenn „Deutschland“ will, dass Muslime sich mit diesem Land identifizieren, muss es eine Basis schaffen, dass sie sich dem Land zugehörig fühlen können. ${ }^{12}$ Hieran wird deutlich, dass es zu Schieflagen, zu misslingender Identität kommt, wenn Muslime etwa Diskriminierung oder strukturelle Nachteile bei ihrer Suche nach Zugehörigkeit zu

\footnotetext{
${ }^{10}$ Vgl. exemplarisch Jürgen Bolten 2007, S. 13.

${ }^{11}$ Manuel Castells 1997, S. 6 ff.

${ }^{12}$ Die Begriffe Identität und Identifikation gehören insofern zusammen, als Identität die Beziehung zwischen Menschen oder Sachverhalten beschreibt, während Identifikation dieser Beziehung die notwendige emotionale Komponente hinzufügt.
} 
Deutschland erfahren. In dem Falle birgt der Identitätsprozess sozialen oder politischen Sprengstoff. Und bei diesen Fragen liegt der Fokus nicht auf der der Integration von Muslimen in die Gesellschaft, sondern vielmehr darauf, ob die Gesellschaft „den“ Islam als Teil ihrer selbst sieht und akzeptiert - und umgekehrt.

Um dies zu klären, bedarf es eines Diskurses zwischen der Mehrheitsbevölkerung und „den“ Muslimen darüber, was Deutschland ausmachen soll, wie sich Bürgersinn und Wertintegration verstehen und welchen identitätsstiftenden Beitrag Religionen dazu leisten können. Denn Identität ist immer konstruiert und bedarf lebenslanger und immer wieder neuer Aushandlungen im sozialen Kontext. ${ }^{13}$ Dort ist auch der Platz, an dem - im Rahmen der Vorgaben des Grundgesetzes - Werte gemeinsam bestimmt werden. Voraussetzung dafür ist es, sich zu vergegenwärtigen, welche Zugehörigkeits- und welche Ausschlusskriterien für „den“ Islam - oder auch von Teilen - bestimmt werden können. ${ }^{14}$ Der Versuch, im Rahmen dieses Beitrags einige Kriterien dafür zu benennen und so $\mathrm{zu}$ beschreiben, dass deutlich wird, dass dem Allgemeinen des Untersuchungstableaus das Spezifische des Untersuchungsgegenstandes hier: des Islam - bereits inhärent ist, ${ }^{15}$ stößt deshalb allenthalben an Grenzen, weil knappe Definitionen, also „Ein-Satz-Formulierungen über Zugehörigkeit immer problematisch (sind), erst recht, wenn es um so heikle Dinge geht wie Religion [...]. Ich bin hoch gespannt auf den theologischen Diskurs innerhalb eines europäischen Islam. “16 Der hier unternommene Versuch vermeidet solche Ein-Satz-Formulierungen, trägt zum Diskurs bei und öffnet ihn zu einem gesellschaftlichen Diskurs, er ersetzt ihn aber nicht.

\footnotetext{
${ }^{13}$ Vgl. Heiner Keupp u. a. 2002.

${ }^{14}$ Es geht dabei darum, Verbundenheit zu erkennen und nicht um eine „strategische Maßnahme zur Einbindung problematischer Gruppen“, wie Werner Schiffauer 1998, S. 418, solche Versuche kritisch beschreibt.

${ }^{15}$ Bundespräsident Joachim Gauck hat dies in seiner Antrittsrede „Gemeinsamkeit der Verschiedenen“ genannt. Dahinter stecke s. E. eine Vorstellung „von Beheimatung nicht durch Geburt, sondern der Bejahung des Ortes und der Normen, die an diesem Ort gelten", vgl. unter: http://nachrichten. t-online.de/joachim-gauck-loest-islam-debatte-aus/id_56849620/index (31.08.2012).

${ }^{16}$ Gauck im ZEIT-Interview am 31.05.2012, unter: http://www.zeit.de/2012/23/Interview-Gauck/seite-4 (31.08.2012).
} 


\section{Empirische Annäherung an „Zugehörigkeit“}

Norbert Posse

Es wird deutlich, dass eine empirische Klärung einer Aussage wie „Der Islam gehört zu Deutschland“ nicht von ihrer implizierten normativen Bedeutung getrennt werden und somit nicht eindeutig mit „stimmt - stimmt nicht“ beantwortet werden kann. Hinter solchen Aussagen stehen verschiedenen theoretische Konstrukte, die zuvor genauer operationalisiert werden müssten. Am Beispiel des zitierten Satzes müssten für eine Prüfung geklärt werden, was mit „der Islam“, was mit „gehört“ und was mit „Deutschland“ gemeint ist. Je eindeutiger die jeweiligen Operationalisierungen sind, desto objektiver, realibler und valider können entsprechende Merkmale/Indikatoren zur Prüfung bestimmt werden.

Wir können hier also nur versuchen, allgemein mögliche Indikatoren für datengestützte Klärungsversuche zu beschreiben, die zumindest einen Teil der oben beschriebenen Facetten in den Blick zu nehmen helfen. Formuliert man solche qualitativen Merkmale zur Bestimmung eines Begriffs, so sollten die Kategorien drei Bedingungen erfüllen: ${ }^{17}$

- Die Kategorien müssen exakt definiert sein (Genauigkeitskriterium)

- Die Kategorien müssen sich gegenseitig ausschließen (Exklusivitäts-Kriterium)

- Die Kategorien müssen das Merkmal erschöpfend beschreiben (Exhaustivitäts-Kriterium).

Bei den folgenden Versuchen gehen wir davon aus, dass es notwendig ist, das Untersuchungstableau zu erweitern und zu verallgemeinern. Nur dann, wenn sich die Indikatoren auch für die Bestimmung verschiedener religiöser Ausrichtungen oder Weltanschauungen eignen, sind sie auch brauchbar für eine Überprüfung eines spezifischen Untersuchungsgegenstandes. Die zu entwickelnden Indikatoren haben nur dann eine Berechtigung, wenn sie auf verschiedene Länder/Regionen anwendbar sind und so trennscharf differenzieren, so dass vorhandene Unterschiede identifiziert werden können.

Wir haben die Indikatoren vier verschiedenen Organisationsprinzipien zugeordnet, innerhalb derer so komplexe und komplizierte sozialpsychologische

\footnotetext{
${ }^{17}$ Vgl. Jürgen Bortz und Nicola Döring 1995, S. 129.
} 
Sachverhalte wie die hier zur Prüfung anstehende „Zugehörigkeit des Islam zu Deutschland“ abgebildet werden können. Um eine objektive, reliable und valide Aussage treffen zu können, ist es unabdingbar, mehrere unterschiedliche Indikatoren zu erfassen, wenn möglich auch mit unterschiedlichen Methoden (Triangulation). Die meisten der hier vorgeschlagenen Indikatoren sind nicht „eineindeutig“, d.h. die zugrundeliegenden theoretischen Konstrukte führen zu jeweils vielen Indikatoren auf der empirischen Ebene, wobei jedes einzelne dieser Merkmale auch mit anderen theoretischen Konstrukten verbunden sein kann. So ist beispielsweise (fast) jeder Muslim oder Jude beschnitten, aber nicht jeder, der beschnitten ist, ist auch Muslim oder Jude.

\subsection{Erscheinungsbild/empirische Realität}

In diesem Organisationsprinzip sind die Indikatoren zusammengefasst, die sich in der unmittelbaren empirischen Realität wiederfinden lassen, die also direkt erfassbar, beobachtbar sind. Weiterhin ist den Operationalisierungen dieser Gruppe von Indikatoren gemeinsam, dass sie non-reaktiv, stationär und zeitlich relativ stabil sind und außerdem eine relativ hohe Inter-Rater-Übereinstimmung ermöglichen.

Die untersuchungsleitende Frage zu diesem Bereich lautet: Lassen sich in einem Land/in einer Region empirisch beobachtbare Merkmale erfassen, die einer spezifischen Denk- oder Glaubensrichtung eindeutig zuzuordnen sind?

\begin{tabular}{|l|l|l|}
\hline Indikatoren & Beispiele & Bemerkung \\
\hline Gebäude & $\begin{array}{l}\text { Hierzu zählen alle Gebäude und Bauwer- } \\
\text { ke, die in ihrer Form und ihrer Bedeutung } \\
\text { einer Glaubensrichtung zuzuordnen sind: } \\
\text { Kirchen, Moscheen, Tempel, Synagogen, } \\
\text { Königssäle, Heiligtümer, Friedhöfe ... }\end{array}$ & $\begin{array}{l}\text { Deutschland ist rückläufig, sie liegt bei } \\
\text { etwa 33.000, die Zahl der von außen er- } \\
\text { kennbaren Moscheen lässt sich nur in et- } \\
\text { wa angeben, sie liegt bei 300, zusätz- } \\
\text { lich gibt es etwa 2.600 Gebetsräume. } \\
\text { Auch nicht alle christlichen Kirchenge- } \\
\text { bäude sind auf den ersten Blick als sol- } \\
\text { che zu erkennen (modernen Neubau- } \\
\text { ten fehlen teilweise stereotype „Kirchen- } \\
\text { merkmale“) - und nicht alle Gebäude, } \\
\text { die wie Kirchen aussehen, sind auch sol- } \\
\text { che. }\end{array}$ \\
\hline
\end{tabular}




\begin{tabular}{|c|c|c|}
\hline Indikatoren & Beispiele & Bemerkung \\
\hline $\begin{array}{l}\text { Denkmäler, } \\
\text { Wegmarken }\end{array}$ & $\begin{array}{l}\text { Hierzu zählen alle baulichen Merkmale, } \\
\text { in denen eine religiöse Symbolik wieder- } \\
\text { gegeben wird, wie beispielsweise Weg- } \\
\text { kreuze, Statuen, Denkmäler von "Heili- } \\
\text { gen“. }\end{array}$ & $\begin{array}{l}\text { Bei der Nutzung dieses Indikators muss } \\
\text { berücksichtigt werden, dass verschiede- } \\
\text { ne Glaubensrichtungen eine figürliche } \\
\text { oder bildliche Darstellung nicht zulas- } \\
\text { sen. }\end{array}$ \\
\hline Symbole & $\begin{array}{l}\text { Mit dieser Klasse von Indikatoren sind } \\
\text { deutlich sichtbar angebrachte Symbole, } \\
\text { die einer Denk- oder Glaubensrichtung } \\
\text { zuzuordnen sind, gemeint. Hierzu ge- } \\
\text { hören: Kreuze, Mondsichel und Stern, } \\
\text { Allah-Kalligraphie, Davidstern, Om, Rad } \\
\text { des Lebens z.B. in/an Geschäften, an } \\
\text { Häuserwänden. }\end{array}$ & $\begin{array}{l}\text { Ob es sich bei den jeweils beobacht- } \\
\text { baren Symbolen um Schmuckelemente } \\
\text { oder Ausdruck einer spezifische Glau- } \\
\text { bensrichtung handelt, kann in der Regel } \\
\text { nur aus dem Kontext erschlossen wer- } \\
\text { den. Daher sollten diese Indikatoren nie } \\
\text { als alleinige Merkmale zu Überprüfun- } \\
\text { gen entsprechender Fragestellungen her- } \\
\text { angezogen werden. }\end{array}$ \\
\hline $\begin{array}{l}\text { Ortsbezeich- } \\
\text { nungen }\end{array}$ & $\begin{array}{l}\text { Ortsnamen, die einen Hinweis auf einen } \\
\text { religiösen Hintergrund geben, z. B. Orts- } \\
\text { bezeichnungen, die den Namen von „Hei- } \\
\text { ligen“ tragen oder auf bedeutsame religi- } \\
\text { öse Ereignisse hinweisen. } \\
\end{array}$ & $\begin{array}{l}\text { Hiermit wird zumindest ein historischer } \\
\text { Hintergrund abgebildet. }\end{array}$ \\
\hline$\ldots$ & & \\
\hline
\end{tabular}

Die Methode der Wahl ist bei dieser Gruppe von Indikatoren die Beobachtung. Die Skala, auf der das Auftreten eines Indikators abgebildet wird, ist nominal (trifft zu - trifft nicht zu, liegt vor - liegt nicht vor), die Häufigkeit des Auftretens wird gemessen.

\subsection{Prägung des Alltags/Festtage}

Die zweite Gruppe von Indikatoren enthält ebenfalls unmittelbar beobachtbare Merkmale, die allerdings nicht im gleichen Maße statisch sind. Sie prägen den Lebensalltag eines Landes. Ihre Zuordnung zu spezifischen Denk- und Glaubensrichtung ist aber in der Regel nur bei genauer Kenntnis möglich.

Die untersuchungsleitende Frage zu diesem Bereich lautet: Gibt es in einem Land beobachtbare Alltagsprägungen, die einer spezifischen Denk- oder Glaubensrichtung eindeutig zuzuordnen sind?

\begin{tabular}{|c|c|c|}
\hline Indikatoren & Beispiele & Bemerkung \\
\hline $\begin{array}{l}\text { Sitten und } \\
\text { Gebräuche }\end{array}$ & $\begin{array}{l}\text { Hierzu gehören alle im Lebensalltag fest } \\
\text { verankerten Sitten und Gebräuche, deren } \\
\text { Ausübung direkt oder indirekt beobacht- } \\
\text { bar sind, wie z. B. Karneval, Fastenzeit/ } \\
\text { Ramadan, Fastenbrechen, Prozessionen, } \\
\text { Wallfahrten... }\end{array}$ & $\begin{array}{l}\text { Einige dieser Gebräuche haben zwar ei- } \\
\text { ne historische Verankerung in einer Glau- } \\
\text { bensrichtung, ihre ursprüngliche religi- } \\
\text { öse Bindung heute aber verloren. }\end{array}$ \\
\hline
\end{tabular}




\begin{tabular}{|l|l|l|}
\hline Indikatoren & Beispiele & Bemerkung \\
\hline Rituale & $\begin{array}{l}\text { Zu dieser Gruppe gehören z. B. Tauf-, Be- } \\
\text { schneidungs- bzw. ",Mündigkeits“-Ritua- } \\
\text { le, Segnungen, rituelle Waschungen usw. }\end{array}$ & $\begin{array}{l}\text { Zu bedenken ist hier, dass einzelne re- } \\
\text { ligiös verankerte Rituale an bestimmte } \\
\text { Zeiten und Orte gebunden sein, d. h. } \\
\text { nicht zu jeder Zeit und an jedem Ort be- } \\
\text { obachtet werden können. }\end{array}$ \\
\hline $\begin{array}{l}\text { Kleidung, } \\
\text { Schmuck }\end{array}$ & $\begin{array}{l}\text { Diese Gruppe von Merkmalen enthält sol- } \\
\text { che, die bei einzelnen Menschen oder } \\
\text { Gruppen von Menschen zu beobachten } \\
\text { sind, z. B.Kopftuch, Turban, Kippa, Souta- } \\
\text { ne, Kollar, Haartracht, Kreuz, Davidstern, } \\
\text { religiöse Motive bei Tattoos... }\end{array}$ & $\begin{array}{l}\text { Während die Kleidung religiöser Wür- } \\
\text { denträger oft eindeutig als solche identi- } \\
\text { fizierbar ist, ist die einer religiösen Über- } \\
\text { zeugung zuzuordnende Garderobe oft } \\
\text { nicht so einfach zu erkennen. Die Be- } \\
\text { obachtung der Merkmale ist häufig nicht } \\
\text { eindeutig mit dem zugrundeliegenden } \\
\text { theoretischen Konstrukt verbunden, da } \\
\text { z. B. auch religiöse Symbole als modi- } \\
\text { sche Accessoire Verwendung finden. }\end{array}$ \\
\hline Ernährung & $\begin{array}{l}\text { Freitags Fisch, Verzicht auf Schweine- } \\
\text { fleisch, Alkohol. }\end{array}$ & $\begin{array}{l}\text { Dieser Indikator ist nicht vollkommen } \\
\text { trennscharf zu „,Sitten und Gebräuche“. }\end{array}$ \\
\hline$\ldots$ & \multicolumn{2}{|l}{} \\
\hline
\end{tabular}

Bei diesen Indikatoren reicht oftmals die Beobachtung als Methode nicht aus, da die Indikatoren nicht eindeutig sind und ihre religiöse Verankerung nicht immer unmittelbar erschlossen werden kann. Für die Beobachtung gelten analoge Regeln des Messens zur ersten Indikatorengruppe. Bei diesen Indikatoren kann es aber sinnvoll sein, die Beobachtung um die Befragungen, in einzelnen Fällen auch um eine Dokumentenanalyse zu ergänzen.

\subsection{Prägung der Gesellschaftsordnung/ des Rechtsempfindens/Institutionalisierung}

Weniger leicht „sichtbar“ im unmittelbaren Sinne sind die in diesem Organisationsprinzip zusammengefassten Indikatoren. Sie prägen zwar in mehr oder weniger hohem Maße den Wertekonsens eines Landes oder das, was in der politischen Debatte als „Leitkultur“ gemeint ist, ${ }^{18}$ sie sind aber in der Regel nicht eindeutig operationalisiert, und der Ursprung ihrer religiösen Verankerung kann von verschiedenen Glaubensrichtungen beansprucht werden. ${ }^{19}$

${ }^{18}$ Den Begriff „Leitkultur“ verwendete Bassam Tibi 2000, dessen Wertekonsens einer „Europäischen Leitkultur" allerdings gerade die Trennung von Religion und Politik beschreibt (Vorrang der Vernunft vor religiöser Offenbarung, Demokratie, die auf der Trennung von Religion und Politik basiert, Pluralismus und Toleranz).

${ }^{19}$ Das hierfür treffendste Beispiel sind die „Zehn Gebote“. 
Die untersuchungsleitende Frage zu diesem Bereich lautet: Gibt es in den rechtsstaatlichen Verordnungen, Gesetzen, Erlassen oder für die Gesellschaft verbindlichen Regelungen Inhalte und Formulierungen, die einer spezifischen Denkoder Glaubensrichtung eindeutig zuzuordnen sind?

\begin{tabular}{|c|c|c|}
\hline Indikatoren & Beispiele & Bemerkung \\
\hline $\begin{array}{l}\text { Gesetzestexte, } \\
\text { Verfassungen }\end{array}$ & $\begin{array}{l}\text { In dieser Gruppe von Indikatoren sind al- } \\
\text { le legislativen Regelungen zusammenge- } \\
\text { fasst, wie allgemeine Gesetze, Verordnun- } \\
\text { gen, Erlasse, aber auch grundlegende wie } \\
\text { das Grundgesetz, Landesverfassungen. }\end{array}$ & \multirow[t]{2}{*}{$\begin{array}{l}\text { Die Rückführung einzelner } \\
\text { Gesetzesaussagen auf ihre religiöse } \\
\text { Verankerung setzt ein intensives } \\
\text { Quellenstudium voraus. }\end{array}$} \\
\hline $\begin{array}{l}\text { Gebote/ } \\
\text { Verbote }\end{array}$ & $\begin{array}{l}\text { Hiermit sind die Regelungen des Zusam- } \\
\text { menlebens gemeint, die nicht als Gesetz } \\
\text { formuliert sind wie beispielsweise Statu- } \\
\text { ten, Regelungen, Rechtsverordnungen, Er- } \\
\text { lasse. }\end{array}$ & \\
\hline $\begin{array}{l}\text { Urteilsbegrün- } \\
\text { dungen }\end{array}$ & $\begin{array}{l}\text { Mit diesem Indikator sind richterliche Be- } \\
\text { gründungen für Urteile gemeint, die einen } \\
\text { expliziten Bezug zu religiösen Überzeu- } \\
\text { gungen aufweisen. }\end{array}$ & \\
\hline $\begin{array}{l}\text { Fest- und Fei- } \\
\text { ertage }\end{array}$ & $\begin{array}{l}\text { Hiermit sind alle Fest- und Feiertage ge- } \\
\text { meint, die gesetzlich geregelt sind und } \\
\text { deren religiöse Verankerung eindeutig ist. } \\
\text { Hierzu gehören "kirchliche“ Feiertage wie } \\
\text { Ostern, Pfingsten, Weihnachten usw., Op- } \\
\text { ferfest, Mevlid, Ashura-Fest, Jom Kippur, } \\
\text { Sukkot, Neujahrsfest usw. }\end{array}$ & $\begin{array}{l}\text { Dieser Indikator könnte auch der vor- } \\
\text { angegangenen Gruppe zugeordnet wer- } \\
\text { den. Er wird dann markiert, wenn ein } \\
\text { entsprechender Festtag „offiziell“ began- } \\
\text { gen wird. }\end{array}$ \\
\hline $\begin{array}{l}\text { Inhalte in Cur- } \\
\text { ricula, Lehr- } \\
\text { plänen und } \\
\text { Lehrbüchern }\end{array}$ & $\begin{array}{l}\text { Hiermit sind die in einer Gesellschaft vor- } \\
\text { geschriebenen sowie die in der (Unter- } \\
\text { richts-)Praxis realisierten Inhalte gemeint. }\end{array}$ & $\begin{array}{l}\text { Die verbindlichen Regelungen im Bil- } \\
\text { dungssystem sind ebenfalls in der La- } \\
\text { ge, Hinweise auf die Zugehörigkeit einer } \\
\text { Denk- und Glaubensrichtung zu geben. }\end{array}$ \\
\hline $\begin{array}{l}\text { Institutionali- } \\
\text { sierte Mit- } \\
\text { gliedschaften }\end{array}$ & $\begin{array}{l}\text { Hiermit sind (festgeschriebene) Vereinba- } \\
\text { rungen gemeint, in denen geregelt wird, } \\
\text { dass Mitglieder bestimmter Glaubensrich- } \\
\text { tungen in eine Institution berufen werden } \\
\text { müssen, z. B. auf kommunaler Ebene die } \\
\text { Beteiligung der katholischen und evan- } \\
\text { gelischen Kirchen im verschiedenen Aus- } \\
\text { schüssen, im Rundfunkrat etc. }\end{array}$ & $\begin{array}{l}\text { Bei diesem Indikator ist zu unterschei- } \\
\text { den, ob die entsprechenden Regelungen } \\
\text { verpflichtend sind oder lediglich Emp- } \\
\text { fehlungscharakter haben. }\end{array}$ \\
\hline & & \\
\hline
\end{tabular}

Die Methode der Wahl ist hierbei die Dokumentenanalyse (Inhaltsanalyse), sie muss gegebenenfalls um Experten-Befragungen ergänzt werden. 


\section{4 Überzeugungen/Kommunikative Validierung}

Die Form der empirischen Sozialforschung, die in Bezug auf ähnliche Fragestellungen am häufigsten zum Einsatz kommt, ist die „kommunikative Validierung“ einer Aussage mit Hilfe einer Befragung („Stimmen Sie zu, dass ...?" usw.).

Die untersuchungsleitende Frage zu diesem Bereich lautet: Stimmen Menschen bestimmten Aussagen, die einer spezifischen Denk- oder Glaubensrichtung eindeutig zuzuordnen sind, mehrheitlich zu?

Alle bisherigen Indikatoren lassen sich auch in Fragen und Aussagen abbilden, mit denen man die Bürgerinnen und Bürger eines Landes befragen kann. Solche Fragen/Aussagen werden bei dichotomen Ja-Nein-Fragen in der Regel eingeleitet mit: „Ist es (Ihrer Meinung nach) richtig, dass ... “ oder bei (ordinalen) Skalenfragen mit: „Wie stark, wie wichtig, ... ist ...? ?. ${ }^{20}$ Werden in diesem Zusammenhang repräsentative Umfragen durchgeführt, lässt sich von den Ergebnissen her auf die Überzeugungen in einer Gesellschaft schließen.

\subsection{Abschließende Bemerkungen}

Im ersten Teil diese Beitrags wurde die Vielfalt und Unschärfe ausgelotet, die ein Begriff wie „Zugehörigkeit“ für eine empirische Überprüfung mit sich bringt. Eine allgemein gültige Zusammenstellung von Indikatoren zur Beantwortung der Frage der Zugehörigkeit einer Glaubensrichtung zu einem Land - ob Islam zu Deutschland oder das Christentum zur Türkei - lässt sich nicht erstellen. Es lassen sich allerdings mit den oben beschriebenen Indikatoren bzw. einer begründeten Auswahl davon an der „empirischen Oberfläche“ quanitfizierbare Abbilder gesellschaftlicher Realitäten zeichnen, aus denen sich Rückschlüsse auf die „zugrundeliegenden theoretischen Konstrukte" ziehen lassen. Diese theoretischen Konstrukte selbst aber unterliegen dem wissenschaftlichen bzw. gesellschaftlichem Diskurs.

\footnotetext{
${ }^{20} \mathrm{Zu}$ den Arten von Fragen siehe ausführlich Peter Atteslander, 2000, insbesondere das Kapitel 4.4.7 „Fragen nach unterschiedlicher Zentralität von Meinungen“, S. $164 \mathrm{ff}$.
} 


\section{Literatur}

Atteslander, Peter, Methoden der empirischen Sozialforschung, Berlin 2000.

Bortz, Jürgen und Döring, Nicola, Forschungsmethoden und Evaluation, Berlin 2. Aufl. 1995.

Castells, Manuel, The Power of Identity, Oxford 1997, Blackwell (Vol. 2 of: The Information Age: Economy, Society, and Culture).

Deutsch, Karl Wolfgang, Nationalism and Social Communication, New York 1953.

Hero, Markus; Krech, Volkhard und Zander, Helmut (Hrsg.), Religiöse Vielfalt in Nordrhein-Westfalen. Empirische Befunde und Perspektiven der Globalisierung vor Ort, Paderborn 2008.

Keupp, Heiner; Ahbe, Thomas; Gmür, Wolfgang und Höfer, Renate (Hrsg.), Identitätskonstruktionen. Das Patchwork der Identitäten in der Spätmoderne, Reinbek bei Hamburg 2002.

Krech, Volkhard, Islam und Integration - 12 Thesen, in: Friedrich-Ebert-Stiftung (Hrsg.), Policy Nr. 30, Migration - Religion - Integration, Berlin 2009.

Reiterer, Albert F., Nation und Imperium. Reflexionen über die politische Organisation der modernen Weltgesellschaft, Innsbruck 2012.

Schiffauer, Werner, Ausbau von Partizipationschancen islamischer Minderheiten als Weg zur Überwindung des islamischen Fundamentalismus?, in: Bielefeldt, Heiner und Heitmeyer, Wilhelm (Hrsg.), Politisierte Religion. Ursachen und Erscheinungsformen des modernen Fundamentalismus, Frankfurt/Main 1998.

Tibi, Bassam, Europa ohne Identität? Die Krise der multikulturellen Gesellschaft - Leitkultur oder Wertebeliebigkeit, München 2000.

\section{Internetquellen}

Bolten, Jürgen, Interkulturelle Kompetenz, herausgegeben von der Landeszentrale für politische Bildung Thüringen, Erfurt 2007, unter: http://www.thueringen.de/imperia/ $\mathrm{md} /$ content/lzt/interkulturellekompetenz.pdf (31.08.2012).

Institut Info GmbH, Umfrage unter Deutsch-Türken, SPIEGEL vom 17.08.2012, unter: http://www.spiegel.de/politik/deutschland/studie-zu-deutsch-tuerken-integrations willen-steigt-religiositaet-auch-a-850429.html (30.09.2012).

Nourbakhsh, Younes, Soziologie des religiösen Staates im Islam und im Christentum. Dissertation Hamburg 2008, unter: http://ediss.sub.uni-hamburg.de/volltexte/2008/3819/ pdf/SOZIOLOGIE.pdf (31.08.2012).

Nation. Die politische Organisation von moderner Gesellschaft, Innsbruck o.J., unter: http://homepage.uibk.ac.at/ c40285/reiterer_texte/pdf/Nation1.pdf (31.08.2012). 
